\title{
Coupling Coefficients for Systems with Two Open Electronic Shells: Transition Metal Ions with $\mathbf{p}^{\mathrm{M}} \mathbf{d}^{\mathrm{N}}$ Configuration
}

\author{
B. N. PLAKHUTIN AND A.V. ARBUZNIKOV \\ Institute of Catalysis, Novosibirsk, 630090, Russia \\ A. B. TROFIMOV \\ Department of Chemistry, Irkutsk State University, Irkutsk, 664003, Russia
}

\begin{abstract}
We derived the necessary conditions to which the vector coupling coefficients (vcc) $a_{m n}$ and $b_{m n}$, describing atomic L,S-multiplets of $p^{M} d^{N}$ and $d^{N} s^{\prime}$ configurations $(1 \leqslant N \leqslant 9,1 \leqslant M \leqslant 5)$, should satisfy. It is shown that for two-open-shell systems under consideration the unknown vec should satisfy not only usual restrictions resulting from the spheric symmetry, but also some additional equation introduced in the present paper in the form of a postulate. vCc obtained were used for the ab initio calculations (by the general SCF coupling operator method) of several transitionmetal atoms and ions with electronic configurations $3 d^{1} 4 p^{1}, 3 p^{4} 3 d^{3}$, and $3 p^{5} 3 d^{3}$. To check the presented theory, we carried out a detailed comparison between these results and analogous data, obtained by the atomic Roothaan-Hartree-Fock method [2]. 1993 John Wiley \& Sons, Inc.
\end{abstract}

\section{Introduction}

The inner electronic levels in molecules detected by ionization are known to be close to the pure atomic ones [1]. Therefore, to interpret the corresponding experimental data (such as X-ray, photoelectron, Auger spectra, etc.), one usually performs quantum chemical calculations for both the molecule under analysis and the corresponding isolated atom, and for their ions as well.

Within the framework of the restricted Hartree-Fock method (RHF), the atoms are calculated by the specialized atomic program [2,3], as the problem on ionized atom calculation is not completely resolved within the framework of general open-shell SCF theory (i.e., in the general coupling operator (GCO) method [4-8]). In particular, there are some difficulties in calculating ionized atoms with two open electronic shells by the Gco method $[9,10]$.

In the present paper, we propose a solution of this problem for atoms and ions with $p^{N_{p} d^{N_{d}}}$ electronic configuration $\left(1 \leqslant N_{p} \leqslant 5,1 \leqslant N_{d} \leqslant 9\right)$. The calculation of such states is of interest, e.g., when interpreting the Auger spectra for transition-metal compounds [1].

However, the major interest of this problem is concentrated on its theoretical aspect. As is known, to calculate an atom or a highly symmetric molecular system 
with a degenerate open electronic shell one should take special measures to coordinate the following:

(a) symmetry of a nuclear frame (of molecule or atom) with the symmetry of one-electron open-shell orbitals, and

(b) symmetry of a nuclear frame with the symmetry of multielectron wave functions, as well.

Problem (a) was resolved by Roothaan [11], who defined the energy functional in the RHF theory to be an average expectation value for all the degenerate total wave functions of the state under consideration.

The second problem (b) arises in molecular open-shell systems of cubic, tetragonal, and icosahedral symmetry as well as in atoms with the open $d$-shell being in their specific ("non-Roothaan". [12]) spectroscopic states. The solution of this problem was obtained in $[10,12-14]$ for the systems with one open degenerate electronic shell (configuration $\gamma^{\mathrm{N}}$ ). In case of more complicated systems with several degenerate open shells, such as transition-metal ions with a $\mathrm{p}^{\mathrm{N}_{\mathrm{p}} \mathrm{d}^{\mathrm{N}_{\mathrm{d}}} \text { configura }}$ tion, this problem was solved only for states that appear isolated by spin multiplicity [9].

To solve this problem in general, we introduced a new equation for the determination of $a_{m n}$ and $b_{m n}$ coupling coefficients [8], characterizing the state and configuration under consideration in GCO method. This equation was introduced as a postulate, in addition to those proposed before [10]. The validity of postulated equation was substantiated by comparing the results of $\mathrm{gco}$ calculations with similar data obtained within the framework of the atomic RoothaanHartree-Fock theory [2]). However, we failed in understanding the physical meaning of this equation.

\section{Possible States and Energy of Atom (ion) with $\mathrm{p}^{\mathrm{N}_{\mathrm{p}}} \mathrm{d}^{\mathrm{N}_{d}}$ Electronic Configuration}

The possible states of an atom with $\mathrm{p}^{\mathrm{N}_{\mathrm{p}}} \mathrm{d}^{\mathrm{N}_{\mathrm{d}}}$ electronic configuration $\left(1 \leqslant N_{p} \leqslant 5,1 \leqslant N_{d} \leqslant 9\right)$ are found via general rules [15] on the basis of corresponding states arising from the configurations $p^{N_{p}}$ and $d^{N_{d}}$. Let $L_{p}$ and $S_{p}$ be the values of orbital and spin angular momenta in the system with $\mathrm{p}^{\mathrm{N}_{\mathrm{p}}}$ configuration, and $L_{d}$ and $S_{d}$ be those for $d^{N_{d}}$ configuration. Then, in the L,S-coupling approximation [15], possible values for total momenta, $L$ and $S$, in the atom with $p^{N_{p}} d^{N_{d}}$ configuration are as follows:

$$
\begin{gathered}
L=L_{p}+L_{d}, L_{p}+L_{d}-1, \ldots,\left|L_{p}-L_{d}\right| \\
S=S_{p}+S_{d}, S_{p}+S_{d}-1, \ldots,\left|S_{p}-S_{d}\right|
\end{gathered}
$$

Configurations of $\mathrm{p}^{\mathrm{N}_{\mathrm{p}}} \mathrm{d}^{\mathrm{N}_{d}}$ type give rise to a large number of states (L, S-multiplets), most of them being multiple ones. For example, in the $\mathrm{p}^{4} \mathrm{~d}^{3}$ configuration 
considered below, the following states are possible:

$$
\begin{aligned}
\mathrm{p}^{4} \mathrm{~d}^{3} \longrightarrow & { }^{6} \mathrm{G},{ }^{6} \mathrm{~F},{ }^{6} \mathrm{D}(2),{ }^{6} \mathrm{P},{ }^{6} \mathrm{~S}, \\
& { }^{4} \mathrm{I},{ }^{4} \mathrm{H}(3),{ }^{4} \mathrm{G}(5),{ }^{4} \mathrm{~F}(8),{ }^{4} \mathrm{D}(8),{ }^{4} \mathrm{P}(7),{ }^{4} \mathrm{~S}(2), \\
& { }^{2} \mathrm{~K},{ }^{,} \mathrm{I}(3),{ }^{2} \mathrm{H}(6),{ }^{2} \mathrm{G}(10),{ }^{2} \mathrm{~F}(12),{ }^{2} \mathrm{D}(13),{ }^{2} \mathrm{P}(9),{ }^{2} \mathrm{~S}(4) .
\end{aligned}
$$

Here, the numbers in parentheses denote the number of states of the given symmetry. As with the simpler case of atoms with $\mathrm{d}^{\mathrm{N}}$ configuration, it is necessary to be beyond the scope of RHF method to provide a variationally correct calculation of the multiple states [15].

The state energy $E\left(L, S, p^{N_{p}} d^{N_{d}}\right)$ may be presented as a sum of items:

$$
E\left(L, S, p^{N_{p} N^{N_{d}}}\right)=E^{\prime}+E_{p p}+E_{d d}+E_{p d},
$$

where $E_{p p}$ is interelectronic repulsion energy within open p-shell, $E_{d d}$ is that for $d$-shell, and $\mathrm{E}_{\mathrm{pd}}$ is interaction energy between the open p-and $d$-shells. $\mathrm{E}^{\prime}$ is the remaining energy, being the same for all the states of the $p^{N_{p}} d^{N_{d}}$ configuration.

If the considered L, S-multiplet of $\mathrm{p}^{\mathrm{N}_{\mathrm{p}}} \mathrm{N}^{\mathrm{N}_{\mathrm{d}}}$ configuration is not a multiple one, it may be easily shown that

$$
\begin{aligned}
& E_{p p}=E_{p p}\left(L, S, p^{N_{p}} d^{N_{d}}\right)=E_{p p}\left(L_{p}, S_{p}, p^{N_{p}}\right), \\
& E_{d d}=E_{d d}\left(L, S, p^{N_{p} d^{N_{d}}}\right)=E_{d d}\left(L_{d}, S_{d}, d^{N_{d}}\right),
\end{aligned}
$$

i.e., for nonmultiple states $L_{p}, S_{p}$ and $L_{d}, S_{d}$ quantities are still the "good" quantum numbers. [In other words, two different nonmultiple $L, S$-multiplets with the same values of $L_{p}, S_{p}, L_{d}$, and $S_{d}$ are different in energy (2) only by $E_{p d}$ term.] Below, we shall consider these states only.

In the atomic theory [15], separate items of Eq. (2) may be expressed in terms of the Slater-Condon parameters:

$$
\begin{gathered}
\mathrm{E}_{\mathrm{pp}}=c_{\mathrm{pp}}^{(0)} \mathrm{F}_{\mathrm{pp}}^{0}+c_{\mathrm{pp}}^{(2)} \mathrm{F}_{\mathrm{pp}}^{2} ; \\
\mathrm{E}_{\mathrm{dd}}=c_{\mathrm{dd}}^{(0)} \mathrm{F}_{\mathrm{dd}}^{0}+c_{d d}^{(2)} \mathrm{F}_{\mathrm{dd}}^{2}+c_{\mathrm{dd}}^{(4)} \mathrm{F}_{\mathrm{dd}}^{4} ; \\
\mathrm{E}_{\mathrm{pd}}=c_{\mathrm{pd}}^{(0)} \mathrm{F}_{\mathrm{pd}}^{0}+c_{\mathrm{pd}}^{(2)} \mathrm{F}_{\mathrm{pd}}^{2}+c_{\mathrm{pd}}^{(1)} \mathrm{G}_{\mathrm{pd}}^{1}+c_{\mathrm{pd}}^{(3)} \mathrm{G}_{\mathrm{pd}}^{3} .
\end{gathered}
$$

The values of coefficients, $c^{(0)}, c^{(1)}, \ldots$, characterizing the considered $\mathrm{L}, \mathrm{S}$-multiplet and configuration, are presented in Slater's monograph (see Vol. II in [15]). Item $E^{\prime}$, including kinetic energy of electrons, electron-core interaction energy, etc., is not worked out in detail in the theory [15] [see Eq. (8) below].

\section{Equations to Determine Vector Coupling Coefficients}

Within the framework of the general coupling operator (GCO) method [4$8,16]$, the energy of atom with $\mathrm{p}^{\mathrm{N}_{\mathrm{p}} \mathrm{d}^{N_{d}}}$ electronic configuration being in the ${ }^{2 S+1} \mathrm{~L}$ state is as follows:

$$
\mathrm{E}\left(\mathrm{L}, \mathrm{S}, \mathrm{p}^{\mathrm{N}_{\mathrm{d}}} \mathrm{N}^{\mathrm{N}_{\mathrm{d}}}\right)=\mathrm{E}^{\prime}+\sum_{m} \sum_{n} f_{m} f_{n}\left(2 a_{m n} J_{m n}-b_{m n} K_{m n}\right),
$$


where the summation is carried out over all open-shell $p$ - and $d$-orbitals: $\{m\}=$ $\{n\}=\{p\} \oplus\{d\}$, and

$$
E^{\prime}=2 \sum_{k} H_{k k}+\sum_{k} \sum_{l}\left(2 J_{k l}-K_{k l}\right)+2 \sum_{m} f_{m}\left\{H_{m m}+\sum_{k}\left(2 J_{k m}-K_{k m}\right)\right\} .
$$

The subscripts $k$ and $l$ are closed-shell orbital numbers; $f_{m}$ is open-shell occupation number, the latter being equal to either $f_{\mathrm{p}}=\mathrm{N}_{\mathrm{p}} / 2 \mathrm{n}_{\mathrm{p}}=\mathrm{N}_{\mathrm{p}} / 6$ or $f_{\mathrm{d}}=\mathrm{N}_{\mathrm{d}} /$ $2 n_{d}=N_{d} / 10$.

The coefficients $a_{m n}$ and $b_{m n}$, called vector coupling coefficients (vcc) [16], define the electronic configuration and the state of the system under consideration. In the case of transition-metal atoms and ions with the open $d$-shell, these coefficients are not constant and depend on the choice of degenerate open-shell orbitals basis set [10]. Below, we shall suppose angular parts of $p$ - and $d$-orbitals to be fixed

$$
\begin{aligned}
& \sigma=\mathrm{d}_{\mathrm{z}^{2}}, \quad \pi=\mathrm{d}_{\mathrm{xz}}, \quad \pi^{\prime}=\mathrm{d}_{\mathrm{yz}}, \quad \delta=\mathrm{d}_{\mathrm{x}^{2}-\mathrm{y}^{2}}, \quad \boldsymbol{\delta}^{\prime}=\mathrm{d}_{\mathrm{xy}} \\
& \mathrm{x}=\mathrm{p}_{\mathrm{x}}, \quad \mathrm{y}=\mathrm{p}_{\mathrm{y}}, \quad \mathrm{z}=\mathrm{p}_{\mathrm{z}} \text {. }
\end{aligned}
$$

To simplify further formulas, introduce the following designation:

$$
Q_{m n}=2 a_{m n} J_{m n}-b_{m n} K_{m n}
$$

then

$$
\mathrm{E}\left(\mathrm{L}, \mathrm{S}, \mathrm{p}^{\mathrm{N}_{\mathrm{p}}} \mathrm{d}^{\mathrm{N}_{\mathrm{d}}}\right)=\mathrm{E}^{\prime}+\sum_{m} \sum_{n} f_{m} f_{n} Q_{m n}
$$

Thus, to calculate atoms and ions with $\mathrm{p}^{\mathrm{N}_{\mathrm{p}}} \mathrm{d}^{\mathrm{N}_{\mathrm{d}}}$ configuration, it is necessary to determine the set of coefficients, $a_{m n}$ and $b_{m n}$ (7), for each spectroscopic state. Taking into consideration $p$ - and $d$-shell degeneracies $\left(n_{p}=3\right.$, and $\left.n_{d}=5\right)$, in order to calculate the ion with $\mathrm{p}^{N_{\mathrm{p}}} \mathrm{d}^{N_{d}}$ electronic configuration, one should determine $2 \times\left(\mathrm{n}_{\mathrm{p}}+\mathrm{n}_{\mathrm{d}}\right)^{2}=128$ unknown coefficients, $a_{m n}$ and $b_{m n}$, which form matrices of the following shape:

$$
\left\|a_{m n}\right\|=\begin{array}{|l|l|}
\hline a_{p p^{\prime}} & a_{p d} \\
\hline a_{d p} & a_{d d^{\prime}} \\
\hline
\end{array}
$$

where $p, p^{\prime}=\mathrm{x}, \mathrm{y}, \mathrm{z}$, and $d, d^{\prime}=\sigma, \pi, \pi^{\prime}, \delta, \delta^{\prime}$.

A general approach to find $a_{m n}$ and $b_{m n}$ coefficients for the atoms with open $d$-shell was proposed in [10]. After being generalized a little, the results [10] may be also used in the considered case for two open shells.

The first equation to determine unknown vcc $a_{m n}$ and $b_{m n}$ resultant from [10] expresses a physical condition that $L, S$-multiplet energy within two different restricted Hartree-Fock methods (i.e., within the atomic theory [15], and in the Gco method [4-8]) should be the same. Omitting the term, $E^{\prime}$, in Eqs. (2) and 
(11), one obtains

$$
\begin{aligned}
\sum_{m} \sum_{n} f_{m} f_{n} Q_{m n} & =f_{\mathrm{p}}^{2} \sum_{p} \sum_{p^{\prime}} Q_{p p^{\prime}}+f_{\mathrm{d}}^{2} \sum_{d} \sum_{d^{\prime}} Q_{d d^{\prime}}+f_{\mathrm{p}} f_{\mathrm{d}} \sum_{p} \sum_{d}\left(Q_{p d}+Q_{d p}\right) \\
& =\mathrm{E}_{\mathrm{pp}}+\mathrm{E}_{\mathrm{dd}}+\mathrm{E}_{\mathrm{pd}},
\end{aligned}
$$

where $E_{p p}, E_{d d}$, and $E_{p d}$ are defined by Eqs. (4)-(6). After substituting Eqs. (10) and (4)-(6) into Eq. (13), the latter couples the unknown vcc $a_{m n}$ and $b_{m n}$ and the known coefficients $\mathrm{c}^{(0)}, \mathrm{c}^{(1)}, \ldots$

The second equation for vCC calculation proposed in [10] expresses a condition for one-electron open-shell orbitals to be degenerate. In case of $p^{\mathrm{N}_{\mathrm{P}}} \mathrm{d}^{\mathrm{N}_{\mathrm{d}}}$ configuration, there are two sets of degenerate open-shell orbitals, i.e.,

$$
\begin{gathered}
\varepsilon_{p}=\varepsilon_{\underline{p}} ; \quad(p, \underline{p}=\mathrm{x}, \mathrm{y}, \mathrm{z}), \\
\varepsilon_{d}=\varepsilon_{\underline{d}} ; \quad\left(d, \underline{d}=\sigma, \pi, \pi^{\prime}, \delta, \delta^{\prime}\right),
\end{gathered}
$$

where $\varepsilon_{p}$ and $\varepsilon_{d}$ are one-electron energies.

In the open-shell SCF theory [4-8], one-electron energies are the eigenvalues of the general coupling operator, $R$ :

$$
R \phi_{m}=\phi_{m} \varepsilon_{m} .
$$

Using a general expression for $R$, derived by Hirao (see Eq. (3.8) in [7]), one obtains the following relationship:

$$
\varepsilon_{\mathrm{m}}=\left\langle\phi_{\mathrm{m}}|R| \phi_{\mathrm{m}}\right\rangle=\left\langle\phi_{\mathrm{m}}\left|F_{\mathrm{m}}\right| \phi_{\mathrm{m}}\right\rangle=f_{m}\left\{H_{m m}+\sum_{k}\left(2 J_{k m}-K_{k m}\right)+\sum_{n} f_{n} Q_{m n}\right\},
$$

where $F_{\mathrm{m}}$ is the Fock operator for orbital $\phi_{\mathrm{m}}$ ([7], Eq. (2.1)). Substituting Eq. (17) into Eqs. (14) and (15) and carrying out necessary transformations [10], we obtain

$$
\begin{gathered}
f_{\mathrm{p}} \sum_{p^{\prime}} Q_{p p^{\prime}}+f_{\mathrm{d}} \sum_{d} Q_{p d}=f_{\mathrm{p}} \sum_{p^{\prime}} Q_{\underline{p} p^{\prime}}+f_{\mathrm{d}} \sum_{d} Q_{\underline{p d}}, \\
\left(\varepsilon_{p}=\varepsilon_{\underline{p}} ; p, \underline{p}, p^{\prime}=\mathrm{x}, \mathrm{y}, \mathrm{z}\right) . \\
f_{\mathrm{p}} \sum_{p} Q_{d p}+f_{\mathrm{d}} \sum_{d^{\prime}} Q_{d d^{\prime}}=f_{\mathrm{p}} \sum_{p} Q_{d p}+f_{\mathrm{d}} \sum_{d^{\prime}} Q_{\underline{d} d^{\prime}}, \\
\left(\varepsilon_{d}=\varepsilon_{d} ; d, \underline{d}, d^{\prime}=\sigma, \pi, \pi^{\prime}, \delta, \delta^{\prime}\right),
\end{gathered}
$$

where designations in parentheses point at the equation origin [based on either condition (14) or (15)].

Thus, within the framework of approach [10], to determine the vcc $a_{m n}$ and $b_{m n}$ in the $\mathrm{p}^{\mathrm{N}_{\mathrm{p}}} \mathrm{d}^{\mathrm{N}_{\mathrm{d}}}$ configuration, three fundamental relationships (13), (18), and (19) are required. Relationship (18) is broken up into two independent equations $\left(\varepsilon_{\mathrm{x}}=\varepsilon_{\mathrm{y}}\right.$ and $\varepsilon_{\mathrm{x}}=\varepsilon_{\mathrm{z}}$ ), and relationship (19), into four equations, by analogy. (The number of independent equations is determined by the inequalities $p<\underline{p}$ and $d<\underline{d}$, correspondingly). Each of these seven equations, in its turn, may be parted into several linearly independent equations (see below). 
4. Factorization of Eqs. (13), (18), and (19)

To derive the equations, connecting the unknown vcc $a_{m n}$ and $b_{m n}$ and the known quantities of $c^{(0)}, c^{(1)}, \ldots$, in the explicit form, it is necessary to express $J_{m n}$ and $K_{m n}$ integrals in Eqs. (10), (13), (18), and (19) in terms of the SlaterCondon parameters [15].

Corresponding expressions were presented in monograph [15] for case of complex AOs, and in monograph [17] for the real ones (the integrals, $J_{p p^{\prime}}, K_{p p^{\prime}}$ and $J_{d d^{\prime \prime}}$, $K_{d d^{\prime}}$, as well as four-indexed ones $\left.\left\langle d \underline{d} \mid d^{\prime} \underline{d}^{\prime}\right\rangle\right)$. Similar expressions for the integrals, $J_{p d}=\langle p p \mid d d\rangle$ and $K_{p d}=\langle p d \mid p d\rangle$, in the real Ao's basis set were obtained in [18]; we present them below. (To simplify the formulas, we use the designations $\left.\bar{F}^{0}=F_{p d}^{0}, F^{2}=F_{p d}^{2}, \ldots\right)$ :

$$
\begin{aligned}
& J_{\mathrm{xo}}=J_{y \sigma}=\mathrm{F}^{0}-\frac{2}{35} \mathrm{~F}^{2} \\
& \mathrm{Jo}=\mathrm{F}^{0}+\frac{4}{35} \mathrm{~F}^{2} \\
& J_{\mathrm{x} \pi}=J_{\mathrm{y} \pi^{\prime}}=J_{z \pi}=J_{z \pi^{\prime}}=J_{\mathrm{x} \delta}=J_{\mathrm{x} \delta}=J_{\mathrm{y} \delta}=J_{\mathrm{y} \delta}=\mathrm{F}^{0}+\frac{2}{35} \mathrm{~F}^{2}, \\
& J_{x \pi^{\prime}}=J_{y \pi}=J_{z \delta}=J_{z \delta^{\prime}}=\mathrm{F}^{0}-\frac{4}{35} \mathrm{~F}^{2} \\
& K_{\mathrm{xo}}=K_{\mathrm{y} \sigma}=\frac{1}{15} \mathrm{G}^{1}+\frac{18}{245} \mathrm{G}^{3} \\
& K_{2 \sigma}=\frac{4}{15} \mathrm{G}^{1}+\frac{27}{245} \mathrm{G}^{3} \\
& K_{\mathrm{x} \pi}=K_{\mathrm{y} \pi^{\prime}}=K_{z \pi}=K_{z \pi^{\prime}}=K_{\mathrm{x} \delta}=K_{\mathrm{x} \delta}=K_{\mathrm{y} \delta}=K_{\mathrm{y} \delta}=\frac{3}{15} \mathrm{G}^{1}+\frac{24}{245} \mathrm{G}^{3}, \\
& K_{\mathrm{x} \pi^{\prime}}=K_{\mathrm{y} \pi}=K_{z \delta}=K_{\mathrm{z} \delta^{\prime}}=\frac{15}{245} \mathrm{G}^{3} .
\end{aligned}
$$

Substituting the values of $Q_{p d}$ from Eqs. (10) and (20) and those of $Q_{p p^{\prime}}$ and $Q_{d d^{\prime}}$ from Ref. [15] into Eq. (13), and leveling separately the coefficients multiplied by $F_{p p}^{0}, F_{p p}^{2}, F_{d d}^{0}, \ldots, G_{p d}^{3}$ both at the left and at the right sides, nine linear nonuniform equations to determine 128 unknowns $a_{m n}, b_{m n}$ are obtained.

Analogous substitution of $Q_{m n}$ values into each of two Eqs. (18) and each of four Eqs. (19) leads to $12=6 \times 2$ and $28=7 \times 4$ linear uniform equations, respectively. [After such substitution, there are 9, 6, and 7 Slater-Condon parameters in Eqs. (13), (18), and (19), respectively.] Thus, there are totally nine nonuniform and 40 uniform linear equations to determine 128 unknowns $a_{m n}$ and $b_{m n}$.

The obtained set of equations is broken up (factorized) into three groups of the equations, with each group containing unknowns from only one block of matrices (12): $\left\{a_{p p^{\prime}}, b_{p p^{\prime}}\right\},\left\{a_{d d^{\prime}}, b_{d d^{\prime}}\right\}$ and $\left\{a_{d p}, b_{d p}, a_{p d}, b_{p d}\right\}$. 


\subsection{Equations for Determining Coefficients $\mathrm{a}_{\mathrm{dd}}$ and $\mathrm{b}_{\mathrm{d} \mathrm{d}^{\prime}}$}

The set of equations containing unknowns $a_{d d}$ and $b_{d d}$ includes three nonuniform equations derived from relationship (13). These three equations may be written as a single equation directly resultant from Eq. (13):

$$
f_{d}^{2} \sum_{d} \sum_{d^{\prime}} Q_{d d^{\prime}}=c_{\mathrm{dd}}^{(0)} F_{\mathrm{dd}}^{0}+c_{\mathrm{dd}}^{(2)} F_{\mathrm{dd}}^{2}+c_{\mathrm{dd}}^{(4)} F_{\mathrm{dd}}^{4}
$$

After substituting Slater-Condon parameters into $Q_{d d^{\prime}}$ expression (10) and leveling separately the coefficients multiplied by $F_{d d}^{0}, F_{d d}^{2}$, and $F_{d d}^{3}$ both at the left and at the right sides of Eq. (21), we derive three linear nonuniform equations coupling unknowns $a_{d d^{\prime}}$ and $b_{d d^{\prime}}$ with known coefficients $c_{\mathrm{dd}}^{(0)}, c_{\mathrm{dd}}^{(2)}$, and $c_{\mathrm{dd}}^{(4)}$.

Corresponding uniform equations to determine $a_{d d^{\prime}}$ and $b_{d d^{\prime}}$ are derived from the condition for one-electron $d$-orbitals degeneracy (19). Twenty-eight equations obtained above from Eq. (19) can be written as two general equations, one of which contains $a_{d d^{\prime}}$ and $b_{d d^{\prime}}$ unknowns only:

$$
\sum_{d^{\prime}} Q_{d d^{\prime}}=\sum_{d^{\prime}} Q_{\underline{d} d^{\prime}} ; \quad\left(\varepsilon_{d}=\varepsilon_{\underline{d}}, d<\underline{d}\right) .
$$

[As above, designations in parentheses point at the equation origin-see Eqs. (18) and (19).] The second equation derived from Eq. (19) and containing $\left\{a_{d p}, b_{d p}\right\}$ unknowns is presented below [see Eq. (27)].

Collecting into the left side of Eq. (22) all its terms and carrying out the above transformations, we obtain $12=3 \times\left(n_{d}-1\right)$ linear uniform equations. Thus, taking into account Eq. (21), one obtains totally $15=3 \times \mathrm{n}_{\mathrm{d}}$ equations to determine $50=2 \times \mathrm{n}_{\mathrm{d}} \times \mathrm{n}_{\mathrm{d}} a_{d d^{\prime}}, b_{d d^{\prime}}$ unknowns.

By direct comparison, one may see that the obtained Eqs. (21) and (22) coincide exactly with Eqs. (9) and (16) in [10] derived to determine vcc for the atom with $\mathrm{d}^{\mathrm{N}}$ configuration. This result means that vcc $a_{d d^{\prime}}$ and $b_{d d^{\prime}}$, obtained for the multiplet $\left(L_{d}, S_{d}, d^{N}\right)$, keep their values for all nonmultiple (nondouble) states $\left(\mathrm{L}, \mathrm{S}, \mathrm{p}^{\mathrm{N}_{\mathrm{p}}} \mathrm{d}^{\mathrm{N}_{\mathrm{d}}}\right.$ ), if $\mathrm{N}_{\mathrm{d}}=\mathrm{N}$, and quantum numbers $\mathrm{L}, \mathrm{S}$ and $\mathrm{L}_{\mathrm{d}}, \mathrm{S}_{\mathrm{d}}$ are coupled by relationships (1).

By analogy, it may be shown that the coefficients $a_{p p^{\prime}}$ and $b_{p p^{\prime}}$ have the same values both for the $\left(L_{p}, S_{p}, p^{N_{p}}\right)$ and for $\left(L, S, p^{N_{p}} d^{N_{d}}\right)$ multiplets. The corresponding equations are presented below without any detailed comments.

\subsection{Equations for Coefficients $\mathrm{a}_{\mathrm{pp}^{\prime}}$ and $\mathrm{b}_{\mathrm{pp}^{\prime}}$}

$$
\begin{gathered}
f_{\mathrm{p}}^{2} \sum_{p} \sum_{p^{\prime}} Q_{p p^{\prime}}=c_{\mathrm{pp}}^{(0)} \mathrm{F}_{\mathrm{pp}}^{0}+c_{\mathrm{pp}}^{(2)} \mathrm{F}_{\mathrm{pp}}^{2} ; \\
\sum_{p^{\prime}} Q_{p p^{\prime}}=\sum_{p^{\prime}} Q_{\underline{p} p^{\prime}} ; \quad\left(\varepsilon_{p}=\varepsilon_{p}, p<\underline{p}\right) .
\end{gathered}
$$

After substituting Slater-Condon parameters into $Q_{p p^{\prime}}$ expression (10) and leveling separately the coefficients multiplied by $\mathrm{F}_{\mathrm{pp}}^{0}$ and $\mathrm{F}_{\mathrm{pp}}^{2}$ both at the left and at 
the right sides of Eqs. (23) and (24), one obtains $6=2 \times n_{p}$ equations to determine $18=2 \times \mathrm{n}_{\mathrm{p}} \times \mathrm{n}_{\mathrm{p}}$ unknowns $a_{p p^{\prime}}$ and $b_{p p^{\prime}}$.

\subsection{Equations for coefficients $\mathrm{a}_{\mathrm{dp}}, \mathrm{b}_{\mathrm{dp}}, \mathrm{a}_{\mathrm{pd}}$, and $\mathrm{b}_{\mathrm{pd}}$}

Subtracting Eqs. (21) and (23) from Eq. (13), we obtain a nonuniform equation to determine the vCC $a_{d p}, b_{d p}, a_{p d}$, and $b_{p d}$, entering off-diagonal blocks of matrices (12):

$$
f_{\mathrm{p}} f_{\mathrm{d}} \sum_{p \mathrm{p}} \sum_{d}\left(Q_{p d}+Q_{d p}\right)=c_{\mathrm{pd}}^{(0)} \mathrm{F}_{\mathrm{pd}}^{0}+c_{\mathrm{pd}}^{(2)} \mathrm{F}_{\mathrm{pd}}^{0}+c_{\mathrm{pd}}^{(1)} \mathrm{G}_{\mathrm{pd}}^{1}+c_{\mathrm{pd}}^{(3)} \mathrm{G}_{\mathrm{pd}}^{3}
$$

By analogy, subtracting Eqs. (24) and (22) from Eqș. (18) and (19), correspondingly, one obtains two uniform equations:

$$
\begin{array}{ll}
\sum_{d} Q_{p d}=\sum_{d} Q_{p d} ; \quad\left(\varepsilon_{p}=\varepsilon_{p}, p<\underline{p}\right), \\
\sum_{p} Q_{d p}=\sum_{p} Q_{d p} ; \quad\left(\varepsilon_{d}=\varepsilon_{d}, d<\underline{d}\right)
\end{array}
$$

Equations (25)-(27) allow the further simplification. Transforming Eq. (25) in the above manner [see Eq. (21) and the corresponding text], we obtain a set of linear nonuniform equations:

$$
\sum_{\mathrm{j}=1}^{\mathrm{M}_{\mathrm{x}}} \lambda_{\mathrm{ij}} x_{\mathrm{j}}=c_{\mathrm{pd}}^{(\mathrm{i}-1)} / f_{\mathrm{p}} f_{\mathrm{d}} ; \quad \mathrm{i}=1,2, \ldots, \mathrm{M}_{\mathrm{scp}}
$$

where $x_{\mathrm{j}}$ are the $a_{d p}, a_{p d}, b_{d p}$, and $b_{p d}$ unknowns, regulated in some definite way; $\mathbf{M}_{\mathbf{x}}$ is number of unknowns; $\lambda_{\mathrm{ij}}$ are numerical coefficients; and $\mathbf{M}_{\mathrm{sc}}$ is number of Slater-Condon parameters (SCP) in Eq. (25). (In the case under consideration, these values are $M_{x}=2 \times 2 \times n_{p} \times n_{d}=60$ and $\left.M_{s c p}=4\right)$.

Similar transformations in Eqs. (26) and (27) lead to the set of uniform equations

$$
\sum_{j=1}^{M_{x}} \lambda_{i j} x_{j}=0 ; \quad i=M_{\text {sCP }}+1, M_{S C P}+2, \ldots, M_{t}
$$

where $M_{t}$ is total number of Eqs. (28) and (29), equal to $M_{t}=M_{\text {sce }} \times\{1+$ $\left.\left(\mathrm{n}_{\mathrm{p}}-1\right)+\left(\mathrm{n}_{\mathrm{d}}-1\right)\right\}=28$. [The number of equations, obtained separately from Eqs. (26) and (27), is equal to $M_{s c p} \times\left(n_{p}-1\right)$ and $M_{s C p} \times\left(n_{d}-1\right)$, respectively.]

Thus, to determine 60 unknown vcc $a_{d p}, b_{d p}, a_{p d}$, and $b_{p d}$, one gets four nonuniform linear equations (28) and 24 uniform ones (29), i.e., there is some arbitrariness in choosing these vcc. Below we shall show that such arbitrariness is of an essential significance. Therefore, we analyze this set of equations and their solutions for different configurations in more detail.

(1) The set of uniform eqs. (29) does not contain the coefficients $c^{(0)}, c^{(2)}, \ldots$, characterizing the state and configuration of the system under consideration. This means Eqs. (29) are valid for all the atoms and ions possessing $\mathrm{p}^{\mathrm{N}_{\mathrm{p}}} \mathrm{d}^{\mathrm{N}_{\mathrm{d}}}$ con- 
figuration with any possible $N_{p}$ and $N_{d}$ values. Due to $\lambda_{i j}$ coefficients rationality [arising on the basis of coefficients rationality in Eqs. (20)], this set of equations may be solved in integers to avoid truncation errors under computer calculations. In the present paper, we use program [10] for analytical solution of uniform eqs. (29) with rectangular $\lambda_{\mathrm{ij}}$ matrices.

(2) Equations like (28) and (29) were considered in [10] first for the atoms with $\mathrm{d}^{\mathrm{N}}$ configuration, where the problem of arbitrariness appears too when choosing vcc $a_{d d^{\prime \prime}}$ and $b_{d d^{*}}$. [In the last case, $\mathrm{M}_{\mathrm{scP}}=3, \mathrm{M}_{\mathrm{t}}=15$, and $\mathrm{M}_{\mathrm{x}}=50-$ see Eqs. (21) and (22).] As was shown in [10], such arbitrariness did not effect physically significant results: total energy, density matrix, etc., did not depend on this arbitrariness as it should be.

The vCc matrices $\left\|a_{d d^{\prime}}\right\|$ and $\left\|b_{d d^{*}}\right\|$, obtained in [10] for various nonmultiple states of $\mathrm{d}^{N}$ configuration, may be divided into two groups, in accordance with the known division of the states into "Roothaan's" and "non-Roothaan's" ones [12-14]. In case of the non-Roothaan states, for which $c_{\mathrm{dd}}^{(2)} \neq c_{\mathrm{dd}}^{(4)}[10]$, at least one of these matrices must be a nonsymmetric one: $\left\|a_{d d d}\right\| \neq\left\|a_{d d d^{\prime}}\right\|^{\mathrm{T}}$ and/or $\left\|b_{d d d^{\prime}}\right\| \neq$ $\left\|b_{d d^{\prime}}\right\|^{T}$, despite a large arbitrariness in choosing vcc $\left(\mathbf{M}_{\mathbf{x}}-\mathbf{M}_{\mathbf{t}}=35\right)$.

On the other hand, in case of the Roothaan-type states, for which $c_{\mathrm{dd}}^{(2)}=c_{\mathrm{dd}}^{(4)}$ [10], there are no restrictions toward the shape $\left\|a_{d d^{\prime}}\right\|$ and $\left\|b_{d d^{\prime}}\right\|$ matrices. In particular, the corresponding vcc may be obtained in the standard Roothaan' form [11]: $a_{d d^{\prime}}=a$, and $b_{d d^{\prime}}=b$, where $a=\left[-7 c_{\mathrm{dd}}^{(2)}+\mathrm{N}(\mathrm{N}-1)\right] / 100 f_{\mathrm{d}}^{2}$ and $b=$ $-7 c_{\mathrm{dd}}^{(2)} / 10 f_{\mathrm{d}}^{2}[10]$.

(3) A similar analysis of Eqs. (28) and (29) for $\mathrm{p}^{\mathrm{N}}$ configuration shows that the arbitrariness in the choice of the vCC $a_{p p^{\prime}}$ and $b_{p p^{\prime}}\left[\mathrm{M}_{x}-\mathrm{M}_{\mathrm{t}}=12\right.$, see Eqs. (23) and (24)] also does not influence the results of quantum chemical calculations. Therefore, below we use the values for these vcc, derived by Roothaan [11].

(4) A quite different situation takes place in case of the vcc $a_{d p}, b_{d p}, a_{p d}$, and $b_{p d}$. Here, a general solution of Eqs. (28) and (29) contains $\mathrm{M}_{\mathrm{x}}-\mathrm{M}_{\mathrm{t}}=32$ arbitrary parameters. Changing these parameters, one may obtain the different sets of vcc for each spectroscopic state.

Using these vCC, we performed the ab initio calculations on the titanium and vanadium atoms and their ions with electronic configurations $3 d^{1} 4 p^{1}$ and $3 p^{5} 3 d^{3}$, $3 p^{4} 3 d^{3}$, respectively. The peculiarities of the calculation scheme and the list of calculated states are presented below.

These calculations revealed a dependence of the calculated results (the total energy, expansion coefficients, one-electron energies $\varepsilon_{p}$, $\varepsilon_{d}$, etc.) on the choice of the arbitrary parameters, within the accuracy with which vcc $a_{d p}, b_{d p}, a_{p d}$, and $b_{p d}$ are determined. [Recall that vcc $\left(a_{d d^{\prime \prime}}, b_{d d^{\prime}}\right)$ and $\left(a_{p p^{\prime}}, b_{p p^{\prime}}\right)$ were taken unchanged from [10] and [11], respectively].

Here it should be noticed that symmetry characteristics of the electronic distribution, such as the proper $p$ - and $d$-shell degeneracy (14) and (15), as well as relationships (20) and [17] between the interelectronic repulsion integrals, were obtained correctly in all the calculations and did not depend on the arbitrary parameters choice.

(5) The analysis of these results led us to the conclusion that the set of equations $\left\{(13),(18)\right.$, and (19)\} to determine vcc $a_{m n}, b_{m n}$ for the atoms and ions with 
$\mathrm{p}^{\mathrm{N}_{\mathrm{p}}} \mathrm{d}^{\mathrm{N}_{\mathrm{d}}}$ configuration is necessary but not sufficient, and some additional equations are required.

\section{Additional Equation to Determine Coefficients $a_{d p}, b_{d p}, a_{p d}$, and $b_{p d}$}

Taking into account the above arguments, an additional equation is needed to determine vcc from the off-diagonal blocks of matrices (12) only. The desirable equation is the following:

$$
\sum_{p} \sum_{d} Q_{p d}=\sum_{p} \sum_{d} Q_{d p}
$$

where, in general, $Q_{p d} \neq Q_{d p}$

The correctness of Eq. (30) is substantiated below by comparing the results of quantum chemical calculations obtained within two different (but equivalent) Hartree-Fock methods, i.e., by the GCo method $[4-8,16]$ and the atomic Roothaan-Hartree-Fock method [2] as well. However, there is not any strict theoretical substantiation of this formula yet, and the latter may be regarded as a certain postulate (see also the discussion in Section 7).

By the above technique, Eq. (30) is transformed into the set of four uniform equations. Thus, finally there are 4 nonuniform linear equations (25) and 28 uniform ones $\{(25)-(27) ; 30)\}$ to determine 60 unknown vcc $a_{d p}, b_{d p} ; a_{p d}$, and $b_{p d}$, and, consequently, 28 additional arbitrary relationships may be used.

$A$ fundamental difference in this solution from that described in the previous section consists in the following: The atomic energy and other physical properties calculated with the use of Eq. (30) do not depend on the choice of 28 arbitrary parameters, as it should be. In all cases, new values of energy (see Table IV) became lower than those obtained in the previous section.

To present the obtained vcc $a_{d p}, a_{p d}, b_{d p}$, and $b_{\rho d}$ in a convenient form, we used the additional "natural" relationships between them:

$$
\begin{array}{llll}
a_{\sigma x}=a_{\sigma y} ; \quad a_{\pi x}=a_{\pi z} ; \quad a_{x y}=a_{\pi \xi} ; & a_{\delta x}=a_{\delta y} ; & a_{\delta x}=a_{\delta y} ; \\
a_{x \pi}=a_{x \delta}=a_{x \delta} ; \quad a_{y \pi^{\prime}}=a_{y \delta}=a_{y \delta} ; & a_{z \pi}=a_{z \pi^{\prime}} ; & a_{z \delta}=a_{z \delta} ;
\end{array}
$$

and similar relationships for the $b_{d p}$ and $b_{p d}$ coefficients $\left(b_{a x}=b_{o y} ; \ldots\right.$; $\left.b_{z \delta}=b_{z 5}\right)$. These relationships follow in a natural way from Eqs. (25)-(27) and (30), until the unknowns $a_{\sigma x}$ and $a_{\sigma y} ; a_{\pi x}$ and $a_{\pi z} ; \ldots$; enter Eqs. (25)-(27) and (30) with the same coefficients. Therefore, the additional relationships (31) and (32) do not change the number of linearly independent equations (32 equations totally), but reduce the number of unknowns from 60 to 38 .

Among these $38 \mathrm{vcc}$, there are only four physically independent ones, in accordance with the number of independent coefficients in Eq. (25). (See the discussion on this problem in [10]). As independent vcc, the following ones were chosen:

$$
\begin{gathered}
a_{\sigma \mathrm{x}}=\left(4 c^{(0)}-35 c^{(2)}\right) / 240 f_{\mathrm{p}} f_{\mathrm{d}}, \\
a_{\sigma z}=\left(4 c^{(0)}+70 c^{(2)}\right) / 240 f_{\mathrm{p}} f_{\mathrm{d}} ; \\
b_{\sigma \mathrm{x}}=\left(27 \times 15 c^{(1)}-4 \times 245 c^{(3)}\right) / 900 f_{\mathrm{p}} f_{\mathrm{d}}, \\
b_{\sigma z}=\left(-36 \times 15 c^{(1)}+2 \times 245 c^{(3)}\right) / 900 f_{\mathrm{p}} f_{\mathrm{d}}
\end{gathered}
$$


where $c^{(0)}=c_{\mathrm{pd}}^{(0)}=\mathrm{N}_{\mathrm{p}} \times \mathrm{N}_{\mathrm{d}} ; c^{(2)}=c_{\mathrm{pd}}^{(2)}, \ldots$ The other coefficients of $a_{d p}$ and $a_{p d}$ type, satisfying Eqs. (25)-(27) and (30), are expressed in terms of independent coefficients (33):

$$
\begin{gathered}
a_{\pi \mathrm{x}}=a_{\pi \mathrm{y} y}=a_{\delta \mathrm{x}}=a_{\delta \mathrm{x}}=\left(a_{\sigma \mathrm{x}}+2 a_{\sigma \mathrm{z}}\right) / 3, \\
a_{\pi \mathrm{y}}=a_{\pi^{\prime} \mathrm{x}}=a_{\delta z}=a_{\delta \mathrm{z}}=\left(4 a_{\sigma \mathrm{x}}-a_{\sigma \mathrm{z}}\right) / 3 ; \\
a_{\mathrm{x} \sigma}=\left(5 a_{\pi \mathrm{y}}-3 A_{1}\right) / 2 \\
a_{\mathrm{x} \pi}=\left(5 a_{\sigma \mathrm{z}}+A_{1}\right) / 6 \\
a_{x \pi^{\prime}}=A_{1} \\
a_{y \sigma}=10 a_{\pi \mathrm{x}}-9 A_{2} \\
a_{y \pi}=-5 a_{\sigma z}+6 A_{2} \\
a_{y \pi^{\prime}}=A_{2} \\
a_{z \sigma}=-5 a_{\pi \mathrm{y}}+6 A_{3} \\
a_{z \pi}=5 a_{\sigma \mathrm{x}}-4 A_{3} \\
a_{z \delta}=A_{3},
\end{gathered}
$$

where $A_{1}, A_{2}$, and $A_{3}$ are some arbitrary parameters. Similar expressions for the $b_{d p}$ and $b_{p d}$ coefficients are derived from formulas (35) and (36) by replacing all $a_{\mathrm{ik}}$ coefficients simultaneously by the corresponding ones $b_{\mathrm{ik}}$ (i.e., $a_{\pi \mathrm{x}}$ is replaced by $b_{\pi x} ; a_{\pi^{\prime} y}$ by $b_{\pi^{\prime} y} ;$ etc.), and also by replacing the arbitrary parameters $A_{1}, A_{2}$, and $A_{3}$ by $B_{1}, B_{2}$, and $B_{3}$, correspondingly.

\section{Vector Coupling Coefficients for $\mathrm{s}^{1} \mathrm{~d}^{\mathrm{N}}$ Configuration}

The approach derived above may be also used to determine vcc for the transition-metal atoms (ions) with $\mathrm{s}^{1} \mathrm{~d}^{\mathrm{N}}$ configuration $(1 \leqslant \mathrm{~N} \leqslant 9)$. The coefficients $a_{d s}, b_{d s}$ and $a_{s d}, b_{s d}$ are defined from the following equations:

$$
\begin{gathered}
f_{\mathrm{s}} f_{\mathrm{d}} \sum_{d}\left(Q_{s d}+Q_{d s}\right)=c_{\mathrm{sd}}^{(0)} \mathrm{F}_{\mathrm{sd}}^{0}+c_{\mathrm{sd}}^{(2)} \mathrm{F}_{\mathrm{sd}}^{2}, \\
Q_{d s}=Q_{\underline{d s}} ; \quad\left(\varepsilon_{d}=\varepsilon_{\underline{d}}, d<\underline{d}\right), \\
\sum_{d} Q_{s d}=\sum_{d} Q_{d s},
\end{gathered}
$$

which are similar to Eqs. (25), (27), and (30), respectively. (The coefficients, $a_{s}$ and $b_{s s}$, from the corresponding diagonal block in matrix (12), are zero: $a_{s s}=$ $\left.b_{s s}=0\right)$. Taking into account that $J_{s d}=\mathrm{F}_{s d}^{0}$ and $K_{s d}=(1 / 5) \mathrm{F}_{s d}^{2}$ [15], and omitting the intermediate elementary transformations, one obtains the following solution of Eqs. (37)-(39):

$$
\begin{gathered}
a_{\sigma \mathrm{s}}=a_{\pi \mathrm{s}}=a_{\pi \mathrm{s}}=a_{\delta \mathrm{s}}=a_{\delta s}=a, \\
b_{\sigma \mathrm{s}}=b_{\pi \mathrm{s}}=b_{\pi \mathrm{s}}=b_{\delta \mathrm{s}}=b_{\delta \mathrm{s}}=b ; \\
\sum_{d} a_{s d}=5 a, \quad \sum_{d} b_{s d}=5 b ;
\end{gathered}
$$

where $a=c_{\mathrm{sd}}^{(0)} / 20 f_{\mathrm{s}} f_{\mathrm{d}}=1$, and $b=-c_{\mathrm{sd}}^{(2)} / f_{\mathrm{d}}$. 
Thus, in the $\mathrm{s}^{1} \mathrm{~d}^{\mathrm{N}}$ configuration, the coefficients, $a_{d s}$ and $b_{d s},\left(d=\sigma, \pi, \pi^{\prime}, \delta, \delta^{\prime}\right)$ are determined uniquely $(40)$ for each state, while $a_{s d}, b_{s d}$ coefficients are determined within the accuracy with some arbitrary relationships [there are only two Eqs. (41) to determine 10 coefficients]. In particular, one may put $a_{s d}=a=1$ and $b_{s d}=b$.

\section{Results of Calculations and Discussion}

By formulas (31)-(36) we calculated the vcc $a_{d p}, b_{d p}, a_{p d}$, and $b_{p d}$ for the set of atomic states arising from the configurations $\mathrm{p}^{5} \mathrm{~d}^{3}, \mathrm{p}^{4} \mathrm{~d}^{3}$, and $\mathrm{p}^{1} \mathrm{~d}^{1}$. The list of states, as well as coefficients $c_{\mathrm{pd}}^{(0)}, c_{\mathrm{pd}}^{(1)}, \ldots$, necessary for calculation, is presented in Table $\mathrm{I}$.

Tables II and III present a general form of the matrices $\left\|a_{d p}\right\|,\left\|b_{d p}\right\|,\left\|a_{p d}\right\|$, and $\left\|b_{p d}\right\|$ satisfying Eqs. (25)-(27) and (30) and additional relationships (32) and (33) for nonmultiple states of the $\mathrm{p}^{\mathrm{M}} \mathrm{d}^{\mathrm{N}}$ configuration.

TABLE I. The coefficients $c_{p d}^{(k)}$ in the expression for $E_{p d}$ energy [see Eqs. (6) and (25)].

\begin{tabular}{|c|c|c|c|c|}
\hline $\begin{array}{c}\text { Configuration, } \\
\text { state }\end{array}$ & $F_{p d}^{0}$ & $F_{p d}^{2}$ & $\mathbf{G}_{\mathrm{pd}}^{1}$ & $G_{p d}^{3}$ \\
\hline$p^{5} d^{3}, G$ & 15 & $1 / 35$ & $-18 / 15$ & $-189 / 245$ \\
\hline & 15 & $-3 / 35$ & $-18 / 15$ & $-144 / 245$ \\
\hline & 15 & $-3 / 35$ & $-18 / 15$ & $-84 / 245$ \\
\hline $\mathrm{p}^{4} \mathrm{~d}^{3},{ }^{6} \mathrm{G}$ & 12 & $\div 1 / 35$ & $-18 / 15$ & $-189 / 245$ \\
\hline & 12 & $3 / 35$ & $-18 / 15$ & $-189 / 245$ \\
\hline${ }^{4} I$ & 12 & $3 / 35$ & $-18 / 15$ & $-129 / 245$ \\
\hline${ }^{2} \mathbf{K}$ & 12 & $-6 / 35$ & $-18 / 15$ & $-69 / 245$ \\
\hline$p^{1} d^{1},{ }^{3} F$ & 1 & $2 / 35$ & $-6 / 15$ & $-3 / 245$ \\
\hline
\end{tabular}

TABLE II. General form of $\left\|a_{p d}\right\|$ and $\left\|b_{p d}\right\|$ matrices, satisfying Eqs. (25)-(27) and (30) and additional relationships (31) and (32) for nonmultiple states of $p^{\mathrm{N}_{\mathrm{p}}} \mathrm{d}^{\mathrm{N}_{\mathrm{d}}}$ configuration. ${ }^{\mathrm{A}}$

\begin{tabular}{|c|c|c|c|}
\hline & $\mathbf{x}$ & y & $\mathbf{z}$ \\
\hline \multicolumn{4}{|l|}{$\left\|a_{p d}\right\|^{T}$ matrix } \\
\hline 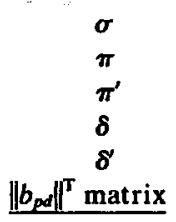 & $\begin{array}{l}\left(5 a_{\text {zy }}-3 A_{1}\right) / 2 \\
\left(5 a_{\sigma 2}+A_{1}\right) / 6 \\
A_{1} \\
\left(5 a_{\sigma 2}+A_{1}\right) / 6 \\
\left(5 a_{\sigma z}+A_{1}\right) / 6\end{array}$ & $\begin{array}{l}10 a_{\pi \mathrm{x}}-9 A_{2} \\
-5 a_{\sigma \mathrm{z}}+6 A_{2} \\
A_{2} \\
A_{2} \\
A_{2}{ }^{\mathrm{b}}\end{array}$ & $\begin{array}{l}-5 a_{\pi y}+6 A_{3} \\
5 a_{\sigma x}-4 A_{3} \\
5 a_{\sigma x}-4 A_{3} \\
A_{3} \\
A_{3}\end{array}$ \\
\hline $\begin{array}{l}\sigma \\
\pi \\
\pi^{\prime} \\
\delta \\
\delta^{\prime}\end{array}$ & $\begin{array}{l}\left(5 b_{\pi y}-3 B_{1}\right) / 2 \\
\left(5 b_{\sigma z}+B_{1}\right) / 6 \\
B_{1} \\
\left(5 b_{\sigma z}+B_{1}\right) / 6 \\
\left(5 b_{\sigma z}+B_{1}\right) / 6\end{array}$ & $\begin{array}{c}10 b_{\pi x}-9 B_{2} \\
-5 b_{a z}+6 B_{2} \\
B_{2} \\
B_{2} \\
B_{2}{ }^{b}\end{array}$ & $\begin{aligned}-5 b_{\pi y}+6 B_{3} \\
5 b_{\sigma x}-4 B_{3} \\
5 b_{\sigma x}-4 B_{3} \\
B_{3} \\
B_{3}\end{aligned}$ \\
\hline
\end{tabular}

${ }^{4}$ See footnotes to Table III.

${ }^{\mathrm{b}} A_{1}, A_{2}, A_{3}$ and $B_{1}, B_{2}, B_{3}$ are arbitrary parameters. 
TABLE III. General form of $\left\|a_{d p}\right\|$ and $\left\|b_{d p}\right\|$ matrices, satisfying Eqs. (25)-(27) and (30) and additional relationships (31) and (32) for nonmultiple states of $p^{N_{p}}{ }^{N_{d}}$ configuration. ${ }^{2}$

\begin{tabular}{|c|c|c|c|c|c|}
\hline & $\boldsymbol{\sigma}$ & $\pi$ & $\pi^{\prime}$ & $\delta$ & $\delta^{\prime}$ \\
\hline \multicolumn{6}{|l|}{$\left\|a_{d p}\right\|^{T}$ matrix } \\
\hline$x$ & $a_{\sigma x}$ & $a_{\pi x}$ & $a_{\pi y}$ & $a_{\pi x}$ & $a_{\pi x}$ \\
\hline$y$ & $a_{\sigma x}^{b}$ & $a_{x y}$ & $a_{\pi x}$ & $a_{\pi x}$ & $a_{\pi}$ \\
\hline$\left\|b_{d p}\right\|^{T}$ matrix & $a_{a z}$ & $a_{\pi x}$ & $a_{\pi x}$ & $a_{\pi y}$ & $a_{m}$ \\
\hline$x$ & $b_{\sigma x}$ & $b_{\pi x}$ & $b_{x y}$ & $b_{\pi \mathrm{x}}$ & $b_{\pi x}$ \\
\hline$y$ & $b_{\sigma x}{ }^{b}$ & $b_{n y}$ & $b_{\pi x}$ & $b_{\pi x}$ & $b_{\pi x}$ \\
\hline$z$ & $b_{\alpha z}$ & $b_{\pi \mathrm{x}}$ & $b_{\pi x}$ & $b_{\pi y}$ & $b_{m}$ \\
\hline
\end{tabular}

'In Tables II and III, we present the transposed matrices $\left\|a_{d p}\right\|^{T},\left\|b_{d p}\right\|^{T},\left\|a_{p d}\right\|^{T}$, and $\left\|b_{p d}\right\|^{T}$. [The definition of the corresponding direct matrices $\left\|a_{d p}\right\|,\left\|b_{d p}\right\|, \ldots$, is given in Eq. (12).] It should be emphasized that $\left\|a_{d p}\right\|^{\mathrm{T}} \neq\left\|a_{\rho d}\right\|,\left\|b_{d p}\right\|^{\mathrm{T}} \neq\left\|b_{p d}\right\|_{\text {, etc.; }}$ see Table II.

${ }^{b}$ Coefficients $a_{\sigma x}, a_{\sigma z}, a_{\pi x}$, and $a_{\pi y}$ and similar coefficients of the $b$-type are determined in Eqs. (33)-(35).

Table IV presents the results of the ab initio calculations on titanium and vanadium atoms and their ions, carried out by the general coupling operator (GCO) method [4-8] with the vCC obtained. The calculations were performed by the MONSTERGAUSS-81 program [19]; the details in calculation scheme were described in [10]. (See also footnote to Table IV.)

TABLE IV. Hartree-Fock energies of vanadium and titanium atoms and their ions ${ }^{\mathrm{a}}$ (calculation by the coupling operator method [4-8]).

\begin{tabular}{|c|c|c|c|}
\hline $\begin{array}{l}\text { Atom } \\
\text { (ion) }\end{array}$ & $\begin{array}{c}\text { Configuration, } \\
\text { state }\end{array}$ & $\begin{array}{c}\text { Origin } \\
\text { of state } \\
\left(L_{d}, S_{d} ; L_{p}, S_{p}\right)\end{array}$ & $\begin{array}{l}\text { Total } \\
\text { energy, } \\
\text { (in au) }\end{array}$ \\
\hline $\mathrm{V}$ & $\begin{array}{ll}3 \mathrm{~d}^{3}, & { }^{4} \mathrm{~F} \\
{ }^{2} \mathrm{H}\end{array}$ & - & $\begin{array}{l}-942.837196 \\
-942.749087\end{array}$ \\
\hline $\mathrm{v}^{+}$ & $\begin{array}{c}3 p^{5} 3 d^{3},{ }^{5} G \\
{ }^{3} I \\
{ }_{1} I\end{array}$ & $\begin{array}{l}{ }^{4} \mathrm{~F}\left(\mathrm{~d}^{3}\right) ;{ }^{2} \mathrm{P}\left(\mathrm{p}^{5}\right) \\
{ }^{2} \mathrm{H}\left(\mathrm{d}^{3}\right) ;{ }^{2} \mathrm{P}\left(\mathrm{p}^{5}\right) \\
{ }^{2} \mathrm{H}\left(\mathrm{d}^{3}\right) ;{ }^{2} \mathrm{P}\left(\mathrm{p}^{5}\right)\end{array}$ & $\begin{array}{l}-941.072849 \\
-940.971360 \\
-940.900879\end{array}$ \\
\hline $\mathrm{V}^{2+}$ & $\begin{array}{r}3 p^{4} 3 d^{3},{ }^{6} G \\
{ }^{6} \mathrm{~F} \\
{ }^{4} \mathrm{I} \\
{ }^{2} \mathrm{~K}\end{array}$ & $\begin{array}{l}{ }^{4} \mathrm{~F}\left(\mathrm{~d}^{3}\right) ;{ }^{3} \mathrm{P}\left(\mathrm{p}^{4}\right) \\
{ }^{4} \mathrm{~F}\left(\mathrm{~d}^{3}\right) ;{ }^{3} \mathrm{P}\left(\mathrm{p}^{4}\right) \\
{ }^{2} \mathrm{H}\left(\mathrm{d}^{3}\right) ;{ }^{3} \mathrm{P}\left(\mathrm{p}^{4}\right) \\
{ }^{2} \mathrm{H}\left(\mathrm{d}^{3}\right) ;{ }^{3} \mathrm{D}\left(\mathrm{p}^{4}\right)\end{array}$ & $\begin{array}{l}-938.748763 \\
-938.702954 \\
-938.531404 \\
-938.450883\end{array}$ \\
\hline $\mathrm{Ti}$ & $\begin{array}{l}3 d^{2},{ }^{3} F \\
3 d^{2} 4 p^{1},{ }^{3} F\end{array}$ & ${ }^{2} \mathrm{D}\left(\mathrm{d}^{\prime}\right) ;{ }^{2} \mathrm{P}\left(\mathrm{p}^{\mathrm{l}}\right)$ & $\begin{array}{l}-848.367900 \\
-845.342161\end{array}$ \\
\hline
\end{tabular}

${ }^{\mathrm{a}}$ The same Gaussian basis set was used to calculate all the states of vanadium atom and its ions (see basis set $(14 \mathrm{~s} 9 \mathrm{p} 5 \mathrm{~d}) /[8 \mathrm{~s} 4 \mathrm{p} 2 \mathrm{~d}]$ with contraction scheme 3 , for vanadium atom in [21, 22]. Similarly, the same Gaussian basis set $[21,22]$ was used for calculation of the ground and excited states of titanium atom. 
Comparison with the Results of Calculations by the Atomic Roothaan-Hartree-Fock Method [2]

For independent checking of the results, presented in Table IV, we also calculated these systems within the framework of the atomic Roothaan-Hartree-Fock theory (expansion method) [2]. The calculations were performed by the Huzinaga' atomic program [20]. As the above presented method to calculate vcc for $\mathrm{p}^{\mathrm{M}} \mathrm{d}^{\mathrm{N}}$ configuration is based on the parent method [10] for $\mathrm{d}^{\mathrm{N}}$ configuration, we checked the results of the Gco calculations [10] as well.

\section{Configuration $d^{N}$}

Table $V$ presents the results of calculations on the first-row transition atoms (from Sc to Cu), carried out by the atomic program [20], as compared to the similar data $[10,21]$; obtained by the cco method, and by the atomic RoothaanHartree Fock method as well. Based on the data presented, one may observe the identity between total atomic energies, obtained by the $\mathrm{GCO}$ method with nonsymmetric vcc $a_{d d^{\prime}}[10]$, and similar results, obtained within the framework of the atomic theory. The comparison between the other calculated values (such as oneelectron energies $\varepsilon_{i}$, expansion coefficients $c_{\mu i}$, etc.) also showed their complete

TABLE V. Energy of the first-row transition atoms (in au), calculated by two different RHF methods (Gaussian basis set (14s9p5d)/[8s4p2d] from Ref. [21 $]^{\mathrm{a}}$ ).

\begin{tabular}{|c|c|c|c|}
\hline \multirow{2}{*}{$\begin{array}{c}\text { Atom, } \\
\text { state } \\
\text { (configuration) }\end{array}$} & \multirow{2}{*}{$\begin{array}{c}\text { SCF coupling } \\
\text { operator method }[4-8] \\
\text { Calculation } \\
{[10]}\end{array}$} & \multicolumn{2}{|c|}{$\begin{array}{l}\text { Atomic Roothaan-Hartree- } \\
\text { Fock theory [2] }\end{array}$} \\
\hline & & $\begin{array}{c}\text { Calculation } \\
\text { [21] }\end{array}$ & Present work \\
\hline $\begin{array}{c}\mathrm{Sc},{ }^{2} \mathrm{D}\left(\mathrm{d}^{1}\right) \\
\mathrm{Ti},{ }^{3} \mathrm{~F}\left(\mathrm{~d}^{2}\right) \\
\mathrm{V},{ }^{4} \mathrm{~F}\left(\mathrm{~d}^{3}\right) \\
{ }^{4} \mathrm{P} \\
{ }^{2} \mathrm{G} \\
{ }^{2} \mathrm{H},{ }^{2} \mathrm{P} \\
{ }^{2} \mathrm{~F} \\
\mathrm{Cr},{ }^{5} \mathrm{D}\left(\mathrm{d}^{4}\right) \\
\mathrm{Mn},{ }^{6} \mathrm{~S}\left(\mathrm{~d}^{5}\right) \\
\mathrm{Fe},{ }^{5} \mathrm{D}\left(\mathrm{d}^{6}\right) \\
\mathrm{Co},{ }^{4} \mathrm{~F}\left(\mathrm{~d}^{7}\right) \\
\mathrm{Ni},{ }^{3} \mathrm{~F}\left(\mathrm{~d}^{8}\right) \\
\mathrm{Cu},{ }^{2} \mathrm{D}\left(\mathrm{d}^{9}\right) \\
{ }^{2} \mathrm{~S}\left(\mathrm{~d}^{10} \mathrm{~s}^{1}\right)\end{array}$ & $\begin{array}{r}-759.705047 \\
-848.367900 \\
-942.837196 \\
-942.771401 \\
-942.770986 \\
-942.749087 \\
-942.683500 \\
-1043.249620 \\
-1149.787155 \\
-1262.350361 \\
-1381.289383 \\
-1506.720591 \\
-1638.786455 \\
-1638.801243\end{array}$ & $\begin{array}{c}-759.7050 \\
-848.3685 \\
-942.8372 \\
- \\
- \\
- \\
- \\
-1043.2497 \\
-1149.7872 \\
-1262.3504 \\
-1381.2895 \\
-1506.7206 \\
-1638.7867 \\
-1638.8015\end{array}$ & $\begin{array}{r}-759.705048 \\
-848.367900 \\
-942.837196 \\
-942.771402 \\
-942.770986 \\
-942.749088 \\
- \\
-1043.249620 \\
-1149.787155 \\
-1262.350360 \\
-1381.289382 \\
-1506.720590 \\
-1638.786455 \\
-1638.801243\end{array}$ \\
\hline
\end{tabular}

"Contraction of the basis set [21]: CONTRACTION 3 for Sc to Fe, CONTRACTION 1 for Co to $\mathrm{Cu}$. 
identity.* Obviously, such coincidence substantiates the validity of the equations [10] used as the starting point in the present paper.

\section{Configuration $p^{M} d^{N}$}

Analogous atomic calculations were carried out for $\mathrm{V}^{2+}$ and $\mathrm{V}^{+}$ions and the $\mathrm{Ti}$ atom with the electronic configuration $\mathrm{p}^{\mathrm{M}} \mathrm{d}^{\mathrm{N}}$. However, while checking the results of the Gco calculations, presented in Table IV, it became necessary to make more curious calculations, because of the following reason: The initial calculation on the ions with $\mathrm{p}^{\mathrm{M}} \mathrm{d}^{\mathrm{N}}$ configuration by the program [20] with the use of the contracted Gaussian basis sets [21] provided the results to be quite different from those obtained above by the Gco method (see Table VI). On the other hand, as was noted above, for the atoms with $\mathrm{d}^{\mathrm{N}}$ configuration, our results coincide completely with the literature ones (see Table V).

To elucidate why in case of $\mathrm{p}^{\mathrm{M}} \mathrm{d}^{\mathrm{N}}$ configuration the results do not match, ${ }^{\dagger}$ we carried out the additional calculations of these systems by the program in [20], using the saturated uncontracted basis sets of Slater' type orbitals (sTo) [23]. Such calculations are known to yield the results, close to the respective HartreeFock limit. Moreover, we carried out also the numerical Hartree-Fock calculations of these systems by Froese-Fischer' program [24].

The analysis of the obtained results, presented in the first, second, and fourth columns of Table VI, reveals an error in the used version of the program [20]. (The states ${ }^{3} I$ and ${ }^{1} I$ of the configuration $3 \mathrm{p}^{5} 3 \mathrm{~d}^{3}$, and ${ }^{2} \mathrm{~K}\left(3 \mathrm{p}^{4} 3 \mathrm{~d}^{3}\right)$, calculated with the use of the contracted Gaussian basis sets [21], turned out to be lower in energy than the respective Hartree-Fock limit.) Our analysis of the program [20] showed that only uncontracted basis sets might be used to calculate interelectronic repulsion between the open $p$ - and $d$-shells. The results of calculations, obtained after required corrections, are presented in the third column of Table VI.

The analysis of all the results, presented in Table VI, shows the data obtained with the use of different basis sets and the results of numerical RHF calculations to be in a proper correspondence in between. This seems to confirm a correct run of the program [20]. The comparison between the results, obtained by the atomic program with the use of the contracted Gaussian basis sets with the respective GCO ones (Table IV), shows their complete identity, as it should be.

Obviously, such coincidence is not a casual one and, therefore, it may be regarded as a proof for the validity of Eq. (30). Keeping it in mind, we present now some arguments to be the starting point for the authors when deducing Eq. (30).

* The coincidence of the expansion coefficients $c_{\mu \text { i }}$, obtained by two different RHF methods, is not, in general, needed. Strictly speaking, the coincidence should take place only for density matrices, $P_{\mu \nu}=\Sigma_{(i)} f_{i} c_{\mu j} c_{\nu i}[11]$. In the case under consideration, the identity of the expansion coefficients is explained by the canonical expression for the general coupling operator, $R$ ([7], Eq. (3.8)), used in [10] to determine the vcc. This canonical expression does not contain any arbitrary shift operators, in contrast to the equivalent expression for $R$, derived in [4].

tWe did not exclude the possibility for the Gco results from Table IV to be erroneous. 
TABLE VI. Hartree-Fock energy (in au) of Ti and V atoms and their ions (Calculations by atomic programs [24] (numerical RHF calculation) and [20] (expansion method)).

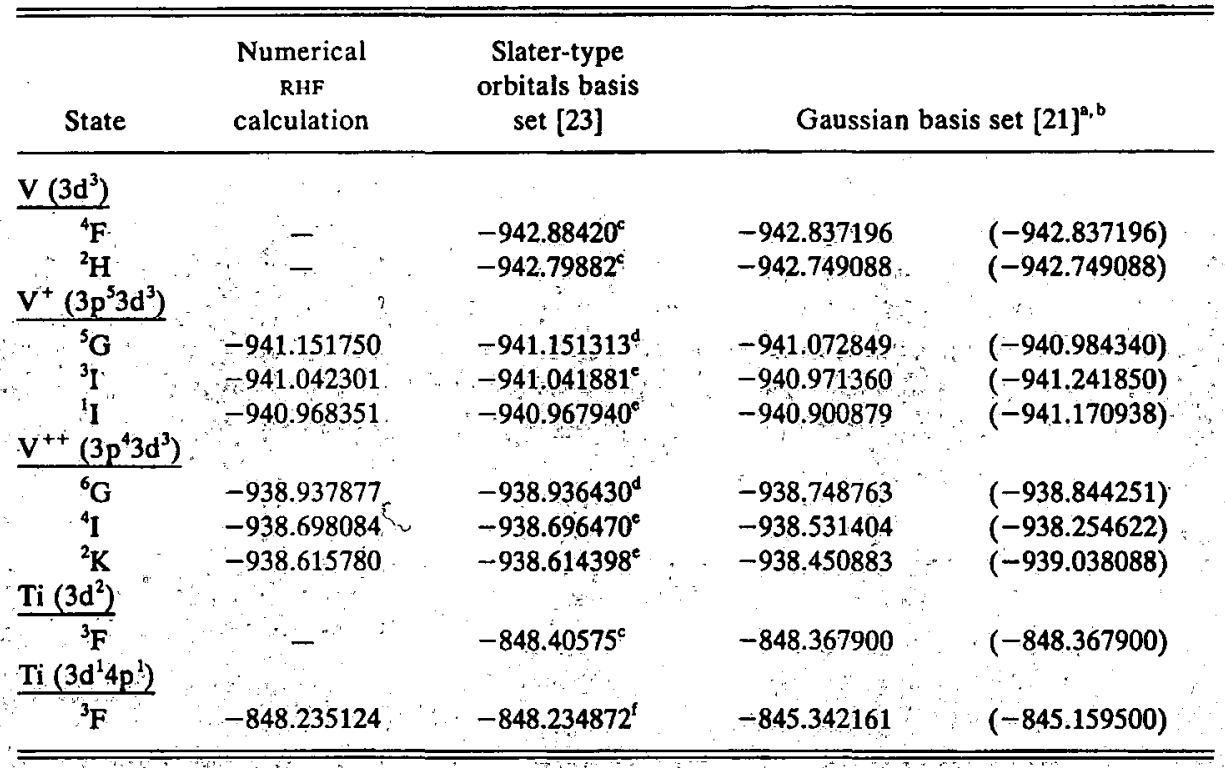

-The same Gaussian basis set [21] was used for all the states of the vanadium atom and its ions (see footnotes to Tables IV and V). Similarly, the same Gaussian basis set [21] was used for both states of titanium atom.

${ }^{b}$ Values in parentheses are results of calculations obtained before revealing an error in program [20] (see discussion in text).

${ }^{c}$ Data from Clementi and Roetti Tables [23]:

d,e Calculation using sto basis set (11s6p5d) [23], optimized for the neutral atom $V\left(3 d^{3}\right)$ in ${ }^{4} F$ and ${ }^{2} \mathrm{H}$ states, respectively.

${ }^{6}$ Calculations using sto basis set [23] for atom $\mathrm{Ti}\left(3 \mathrm{~d}^{2},{ }^{3} \mathrm{~F}\right)$, added by four $4 \mathrm{p}$-exponents from the basis set [23] for atom $\mathrm{Ga}\left(4 \mathrm{p}^{1},{ }^{2} \mathrm{P}\right)$, to describe a $4 \mathrm{p}$-shell of the excited titanium atom.

It should be emphasized that the argumentation to be presented is not of a mathematical strictness, but just points at this equation origin.

In the previous Section 4.3, one mentioned the dependence of one-electron energies, $\varepsilon_{p}$ and $\varepsilon_{d}$, as well as the total energy and other SCF characteristics, on the choice of the arbitrary parameters. Our analysis showed it to be necessary to put an additional restriction on vcc $a_{d p}, b_{d p}, a_{p d}$, and $b_{p d}$ in order to exclude the dependence of one-electron energies, $\varepsilon_{p}$ and $\varepsilon_{d}$, on the arbitrary parameters choice. By Eq. (17), $\varepsilon_{p}$ and $\varepsilon_{d}$ quantities may be presented as follows:

$$
\begin{gathered}
\varepsilon_{p}=f_{\mathrm{p}} H_{p p}+\varepsilon_{p}(\text { closed })+\varepsilon_{p}\left(p^{\prime}\right)+\varepsilon_{p}(d) \\
\varepsilon_{d}=f_{\mathrm{d}} H_{d d}+\varepsilon_{d}(\text { closed })+\varepsilon_{d}\left(d^{\prime}\right)+\varepsilon_{d}(p) .
\end{gathered}
$$

Taking into account Eqs. (25)-(27), it may be shown that the requirement of the independence of $\varepsilon_{p}$ and $\varepsilon_{d}$ on the arbitrary parameters choice is equivalent to 
the relationship

$$
\sum_{p} \varepsilon_{p}(d)=\sum_{d} \varepsilon_{d}(p)
$$

which leads directly to Eq. (30).

\section{Appendix}

After this paper was completed, the authors succeeded in obtaining a mathematical proof of Eq. (30a). Following the referee's recommendation, we included a brief derivation of Eq. (30a) into present paper; a more detailed discussion of some questions arising in this problem will be published elsewhere [25].

First of all, it is necessary to give some notes concerning the different formulations of the SCF coupling operator method. To calculate two-open-shell systems such as transition-metal ions with a configuration $p^{M} d^{N}$ by the SCF coupling operator method, one may use two different formulations of this theory, i.e., the theory $[4-8,16]$, based on the energy functional (7), and a more general theory $[7,8]$ in which the following form of the energy functional is used ([8], Eq. (1-II)):

$$
\mathrm{E}=\sum_{\mathrm{i}} \sum_{\mathrm{j}}\left[\omega_{\mathrm{ij}} H_{\mathrm{ij}}+\sum_{\mathrm{k}} \sum_{l}\left(\alpha_{\mathrm{ij}, \mathrm{k}}\langle\mathrm{ij} \mid \mathrm{kl}\rangle-\beta_{\mathrm{ij}, \mathrm{kl}}\langle\mathrm{i} \mathrm{il} \mid \mathrm{kj}\rangle\right)\right]
$$

where $\alpha_{\mathrm{ij}, \mathrm{kl}}$ and $\beta_{\mathrm{ij}, \mathrm{kl}}$ are the generalized supermatrices of the vCC and $\langle\mathrm{ij} \mid \mathrm{kl}\rangle$ are the four-indexed integrals:

$$
\langle i j \mid k l\rangle=\int \phi_{i}^{*}(1) \phi_{k}^{*}(2)\left(1 / r_{12}\right) \phi_{j}(1) \phi_{i}(2) d V_{1} d V_{2}
$$

(In this section, we use the indices $\mathrm{i}, \mathrm{j}, \mathbf{k}$, and $\mathbf{l}$ for referring to all the occupied orbitals.)

For the most of the open-shell systems, the expression (A-1) may be reduced to a more simple formula (7), containing only two-indexed integrals (Coulomb and exchange) $[8-10,14,16,26]$. Such a reduction is useful since it permits one to avoid using the four-dimensional supermatrices of the vcc and permits one to use existing open-shell quantum chemical programs. If such a reduction is possible in principle, then two considered approaches are equivalent, i.e., the matrices of the SCF coupling operators within these approaches are equal $[7,8]$.

We wish to show that equations of type (30a) follow directly from the variational principle applied to the energy functional of a general form (A-1). Application of the variational principle results in the Euler equations [7,8]:

$$
\sum_{\mathbf{k}} \hat{F}_{\mathrm{ik}}\left|\phi_{\mathrm{k}}\right\rangle=\sum_{\mathrm{i}}\left|\phi_{\mathrm{j}}\right\rangle \theta_{\mathrm{ji}}, \quad \boldsymbol{\theta}_{\mathrm{ji}}=\theta_{\mathrm{ij}}^{*},
$$

where $\theta_{\mathrm{ji}}$ are the Lagrangian multipliers ensuring the orthonormality of the orbitals $\left\langle\phi_{\mathrm{i}} \mid \phi_{\mathrm{j}}\right\rangle=\delta_{\mathrm{ij}}$, and $\hat{F}_{\mathrm{ij}}$ are the Hermitian Fock operators [8]:

$$
\begin{aligned}
& \hat{F}_{\mathrm{ij}}=\left(\omega_{\mathrm{ij}} / 2\right) \hat{h}+\sum_{\mathrm{k}} \sum_{1}\left(A_{\mathrm{ij}, \mathrm{kl}} \hat{J}_{\mathrm{kl}}-B_{\mathrm{ij}, \mathrm{kl}} \hat{K}_{\mathrm{kl}}\right), \\
& A_{\mathrm{ij}, \mathrm{kl}}=\left(\alpha_{\mathrm{ij}, \mathrm{kl}}+\alpha_{\mathrm{kl}, \mathrm{ij}}\right) / 2, \\
& B_{\mathrm{ij}, \mathrm{kl}}=\left(\beta_{\mathrm{ij}, \mathrm{kl}}+\beta_{\mathrm{kl}, \mathrm{ij}}\right) / 2 .
\end{aligned}
$$


[Equation (A-4) coincides with Eq. (7-II) of [8] if one puts $\alpha_{\mathrm{ij}, \mathrm{kl}}=\alpha_{\mathrm{k}, \mathrm{ij}}$ and $\beta_{\mathrm{ij}, \mathrm{kl},}=\beta_{\mathrm{kl}, \mathrm{ij}} *^{*}$

The general Coulomb $\hat{J}_{\mathrm{kl}}$ and exchange $\hat{K}_{\mathrm{kl}}$ operators entering Eq. (A-4) are defined as follows [8]:

$$
\begin{aligned}
& \hat{J}_{\mathrm{k} !}(1) \phi(1)=\left[\int \phi_{k}^{*}(2) \phi_{1}(2)\left(1 / \mathrm{r}_{12}\right) d V_{2}\right] \phi(1), \\
& \hat{K}_{\mathrm{kl}}(1) \phi(1)=\left[\int \phi_{k}^{*}(2) \phi(2)\left(1 / r_{12}\right) d V_{2}\right] \phi_{1}(1)
\end{aligned}
$$

The definition of the general coupling operator $\hat{R}$ valid for all cases for which the Euler equations have the form of Eq. (A-3) was derived by Hirao ([7], Eq. (5.9)). In the definition, this coupling operator satisfies the Hartree-Fock equations ([7], Eq. (5-10))

$$
\begin{gathered}
\hat{R}\left|\phi_{i}\right\rangle=\left|\phi_{\mathrm{i}}\right\rangle \varepsilon_{\mathrm{i}}, \\
\varepsilon_{\mathrm{i}}=\left\langle\phi_{\mathrm{i}}\left|\sum_{\mathrm{k}} \hat{F}_{\mathrm{ik}}\right| \phi_{\mathrm{k}}\right\rangle
\end{gathered}
$$

Thus, to prove the above formula (30a) using the general equation (A-7) for the eigenvalues $\varepsilon_{i}$ of the sCF coupling operator, it is necessary to concretize Eq. (A-4) for the system under consideration.

Let us consider the open-shell electronic configuration $\boldsymbol{\gamma}_{1}^{\mathrm{N}_{1}} \boldsymbol{\gamma}_{\mathrm{II}}^{\mathrm{N}_{11}}$ where $\boldsymbol{\gamma}_{1}$ is the symmetry (irreducible representation) of degenerate orbitals $\left\{\phi_{\eta}\right\}$, and $\gamma_{I I}$ is that for the degenerate orbitals $\left\{\phi_{\eta_{I I}}\right\}$, and $\gamma_{I} \neq \gamma_{I I}$. Without loss of the generality, we omit the closed-shell subsystem. In such a configuration, there are following nonvanishing four-indexed integrals: $\left\langle p q \mid p^{\prime} q^{\prime}\right\rangle,\left\langle m n \mid m^{\prime} n^{\prime}\right\rangle,\langle p q \mid m n\rangle$, and $\langle p m \mid q n\rangle$. (In referring to the individual orbitals, we use the indices $p, q, p^{\prime}, q^{\prime}$ for orbitals of the symmetry $\gamma_{\mathrm{I}} ; \mathrm{m}, \mathrm{n}, \mathrm{m}^{\prime}$, and $\mathrm{n}^{\prime}$ for orbitals of the symmetry $\gamma_{\mathrm{II}}$, and $\mathrm{i}, \mathrm{j}, \mathrm{k}$, and 1 for orbitals of either set.)

In these notations, the Fock operators $\hat{F}_{\mathrm{pq}}, \hat{F}_{\mathrm{pm}}$, and $\hat{F}_{\mathrm{mp}}, \hat{F}_{\mathrm{mn}}$ necessary for the calculation of the degenerate eigenvalues $\varepsilon_{\mathrm{p}}$ and $\varepsilon_{\mathrm{m}}$, correspondingly, have the form

$$
\begin{aligned}
\hat{F}_{\mathrm{pq}}= & \left(\omega_{\mathrm{pq}} / 2\right) \hat{h}+\hat{G}_{\mathrm{pq}}\left(\mathrm{p}^{\prime} \mathrm{q}^{\prime}\right)+\hat{G}_{\mathrm{pq}}(\mathrm{mn}), \\
& \hat{F}_{\mathrm{pm}}=\hat{G}_{\mathrm{pm}}(\mathrm{nq})+\hat{G}_{\mathrm{pm}}(\mathrm{qn}), \\
& \hat{F}_{\mathrm{mp}}=\hat{G}_{\mathrm{mp}}(\mathrm{nq})+\hat{G}_{\mathrm{mp}}(\mathrm{qn}), \\
\hat{F}_{\mathrm{mn}}= & \left(\omega_{\mathrm{mn}} / 2\right) \hat{h}+\hat{G}_{\mathrm{mn}}\left(\mathrm{m}^{\prime} \mathrm{n}^{\prime}\right)+\hat{G}_{\mathrm{mn}}(\mathrm{pq}),
\end{aligned}
$$

where

$$
\hat{G}_{\mathrm{ij}}(\mathrm{kl})=\sum_{\mathrm{k}} \sum_{1}\left(A_{\mathrm{ij}, \mathrm{k} \mathrm{l}} \hat{J}_{\mathrm{kl}}-B_{\mathrm{ij}, \mathrm{k} \mathrm{K}} \hat{K}_{\mathrm{k} \mathrm{l}}\right)
$$

\footnotetext{
"A discussion of the coupling coefficients symmetry is presented in [25].
} 
The Fock operators (A-8) do not contain any other operators of the $\hat{G}_{\mathrm{ij}}$ type, such as $\hat{G}_{\mathrm{pq}}\left(\mathrm{p}^{\prime} \mathrm{m}\right)$ and $\hat{G}_{\mathrm{pm}}\left(\mathrm{m}^{\prime} \mathrm{n}\right)$, since the matrix elements of the latter are equal to zero. For similar reasons, we omit for simplicity the one-electron part $\hat{h}$ of the Fock operators $\hat{F}_{\mathrm{pm}}$ and $\hat{F}_{\mathrm{mp}}$, since to calculate $\varepsilon_{\mathrm{p}}$ and $\varepsilon_{\mathrm{m}}$ by Eq. (A-7), one needs only $\left\langle\phi_{\mathrm{p}}\left|\hat{F}_{\mathrm{pm}}\right| \phi_{\mathrm{m}}\right\rangle$ and $\left\langle\phi_{\mathrm{m}}\left|\hat{F}_{\mathrm{mp}}\right| \phi_{\mathrm{p}}\right\rangle$ matrix elements.

From Eqs. (A-7) and (A-8), it follows that the degenerate eigenvalues $\varepsilon_{\mathrm{p}}$ of open-shell $\gamma_{\mathrm{I}}$ and similar values $\varepsilon_{\mathrm{m}}$ of $\gamma_{\mathrm{II}}$ may be presented as

$$
\begin{gathered}
\varepsilon_{\mathrm{p}}=\left(\omega_{\mathrm{pp}} / 2\right) H_{\mathrm{pp}}+\varepsilon_{\mathrm{p}}(\mathrm{I}, \mathrm{I})+\varepsilon_{\mathrm{p}}(\mathrm{I}, \mathrm{II}), \\
\varepsilon_{\mathrm{m}}=\left(\omega_{\mathrm{mm}} / 2\right) H_{\mathrm{mm}}+\varepsilon_{\mathrm{m}}(\mathrm{II}, \mathrm{II})+\varepsilon_{\mathrm{m}}(\mathrm{II}, \mathrm{I}),
\end{gathered}
$$

where $\varepsilon_{\mathrm{p}}(\mathrm{I}, \mathrm{I})$ is a part of the one-electron energy $\varepsilon_{\mathrm{p}}$ corresponding to the mutual electronic repulsion within the open $\gamma_{1}$ shell and $\varepsilon_{\mathrm{p}}(\mathrm{I}, \mathrm{II})$ is a part of the $\varepsilon_{\mathrm{p}}$ arising due to the coupling between open electronic shells $\gamma_{1}$ and $\gamma_{\mathrm{II}}$. The notations of the individual terms in Eqs. (A-10) are similar to those in Eq. (42). In these terms, the above Eq. (30-a) to be proved may be expressed

$$
\sum_{\mathrm{p}} \varepsilon_{\mathrm{p}}(\mathrm{I}, \mathrm{II})=\sum_{\mathrm{m}} \varepsilon_{\mathrm{m}}(\mathrm{II}, \mathrm{I})
$$

The left and the right sides of Eq. (A-11) are equal to

$$
\begin{aligned}
& \sum_{\mathrm{p}} \varepsilon_{\mathrm{p}}(\mathrm{I}, \mathrm{II})=\sum_{\mathrm{p}} \sum_{\mathrm{q}}\left\langle\phi_{\mathrm{p}}\left|\hat{G}_{\mathrm{pq}}(\mathrm{mn})\right| \phi_{\mathrm{q}}\right\rangle+\sum_{\mathrm{p}} \sum_{\mathrm{m}}\left\langle\phi_{\mathrm{p}}\left|\hat{F}_{\mathrm{pm}}\right| \phi_{\mathrm{m}}\right\rangle, \\
& \sum_{\mathrm{m}} \varepsilon_{\mathrm{m}}(\mathrm{II}, \mathrm{I})=\sum_{\mathrm{m}} \sum_{\mathrm{n}}\left\langle\phi_{\mathrm{m}}\left|\hat{G}_{\mathrm{mn}}(\mathrm{pq})\right| \phi_{\mathrm{n}}\right\rangle+\sum_{\mathrm{m}} \sum_{\mathrm{p}}\left\langle\phi_{\mathrm{m}}\left|\hat{F}_{\mathrm{mp}}\right| \phi_{\mathrm{p}}\right\rangle,
\end{aligned}
$$

correspondingly. Comparing the right sides of Eqs. (A-12) and (A-13), one can see that their first terms are equal to each other since

$$
\left.\left.\left\langle\phi_{\mathrm{p}}\right| A_{\mathrm{pq}, \mathrm{mn}} \hat{J}_{\mathrm{mn}}-B_{\mathrm{pq}, \mathrm{mn}} \hat{K}_{\mathrm{mn}}\right)\left|\phi_{\mathrm{q}}\right\rangle=\left\langle\phi_{\mathrm{m}}\right| A_{\mathrm{mn}, \mathrm{pq}} \hat{J}_{\mathrm{pq}}-B_{\mathrm{mn}, \mathrm{pq}} \hat{K}_{\mathrm{pq}}\right)\left|\phi_{\mathrm{n}}\right\rangle .
$$

Therefore, to prove Eq. (A-11), we should prove the equality

$$
\sum_{\mathrm{p}} \sum_{\mathrm{m}}\left\langle\phi_{\mathrm{p}}\left|\hat{F}_{\mathrm{pm}}\right| \phi_{\mathrm{m}}\right\rangle=\sum_{\mathrm{m}} \sum_{\mathrm{p}}\left\langle\phi_{\mathrm{m}}\left|\hat{F}_{\mathrm{mp}}\right| \phi_{\mathrm{p}}\right\rangle
$$

where

$$
\left\langle\phi_{\mathrm{p}}\left|\hat{F}_{\mathrm{pm}}\right| \phi_{\mathrm{m}}\right\rangle=\left\langle\phi_{\mathrm{p}}\left|\hat{F}_{\mathrm{mp}}^{+}\right| \phi_{\mathrm{m}}\right\rangle=\left\langle\phi_{\mathrm{m}}\left|\hat{F}_{\mathrm{mp}}\right| \phi_{\mathrm{p}}\right\rangle^{*}
$$

[Eq. (A-16) follows from the condition of the Hermiticity of the Fock operators $\hat{F}_{\mathrm{pm}}^{+}=\hat{F}_{\mathrm{mp}},[8]$.] 
Keeping in mind Eq. (A-16), let us show that the sums of matrix elements in both left and right sides of Eq. (A-15) are real values. The left side of Eq. (A-15) may be presented as

$$
\sum_{\mathrm{p}} \sum_{\mathrm{m}}\left\langle\phi_{\mathrm{p}}\left|\hat{F}_{\mathrm{pm}}\right| \phi_{\mathrm{m}}\right\rangle=\sum_{\mathrm{p}} \sum_{\mathrm{k}}\left\langle\phi_{\mathrm{p}}\left|\hat{F}_{\mathrm{pk}}\right| \phi_{\mathrm{k}}\right\rangle-\sum_{\mathrm{p}} \sum_{\mathrm{q}}\left\langle\phi_{\mathrm{p}}\left|\hat{F}_{\mathrm{pq}}\right| \phi_{\mathrm{q}}\right\rangle
$$

where both the terms in right side of Eq. (A-17) are real values, since

$$
\begin{gathered}
\sum_{\mathrm{p}} \sum_{\mathbf{k}}\left\langle\phi_{\mathrm{p}}\left|\hat{F}_{\mathrm{pk}}\right| \phi_{\mathrm{k}}\right\rangle=\sum_{\mathrm{p}} \theta_{\mathrm{pp}}, \\
\sum_{\mathrm{p}} \sum_{\mathrm{q}}\left\langle\phi_{\mathrm{p}}\left|\hat{F}_{\mathrm{pq}}\right| \phi_{\mathrm{q}}\right\rangle=\sum_{\mathrm{p}} \sum_{\mathrm{q}}\left\langle\phi_{\mathrm{q}}\left|\hat{F}_{\mathrm{qp}}\right| \phi_{\mathrm{p}}\right\rangle=\sum_{\mathrm{p}} \sum_{\mathrm{q}}\left\langle\phi_{\mathrm{p}}\left|\hat{F}_{\mathrm{pq}}\right| \phi_{\mathrm{q}}\right\rangle^{*}
\end{gathered}
$$

$\left[\theta_{\mathrm{pp}}\right.$ is a diagonal Lagrangian multiplier (see Eq. (A-3). $]$ Therefore, the sum of the complex matrix elements in the left side of Eq. (A-15) is the real value, and, consequently, the left and the right sides of Eq. (A-15) are equal. Taking into account Eqs. (A-14) and (A-15), one may conclude that the right sides of Eqs. (A-12) and (A-13) are equal to each other. The latter is the proof of Eqs: (A-11) and (30-a).

\section{Acknowledgments}

We would like to thank Prof. A. I. Dement'ev who directed our attention to this problem. Also, the authors wish to thank Prof. I.V. Abarenkov and Prof. G. M. Zhidomirov for the valuable discussions of the paper.

\section{Bibliography}

[1] F. P. Larkins, J. Electron Spectrosc. Rclated Phenom. 51, 115 (1990).

[2] C. C. J. Roothaan and P.S. Bagus, Methods in Computational Physics (Academic Press, New York, 1963), Vol. 2, pp. 47-95.

[3] B. Roos, C. Salez, A. Veillard, and E. Clementi, A General Program for Calculation of Atomic SCF Orbitals by the Expansion Method, Technical Report RJ-518, IBM Research (1968).

[4] G. G. Dyadyusha and V.A. Kuprievich, Theor. Exp. Chem. USSR 7, 729 (1965) (English translation).

[5] S. Huzinaga, J. Chem. Phys. 51, 3971 (1969).

[6] K. Hirao and H. Nakatsuji, J. Chem. Phys. 59, 1457 (1973).

[7] K. Hirao, J. Chem. Phys. 60, 3215 (1974).

[8] R. Carbo and J.M. Riera, A General SCF Theory, Lecture Notes in Chemistry, Vol. 5 (Springer-Verlag, Berlin, 1978).

[9] LI. Domingo and J. I. Burgos, Stud. Phys. Theor. Chem. 62, 103 (1989).

[10] B. N. Plakhutin, G. M. Zhidomirov, and A.V. Arbuznikov, Int. J. Quantum Chem. 41, 311 (1992).

[11] C. C. J. Roothaan, Rev. Mod. Phys. 32, 179 (1960).

[12] B. N. Plakhutin and G. M. Zhidomirov, J. Struct. Chem. USSR 27, 179 (1986) (English translation).

[13] B. N. Plakhutin, G.T. Klimko, M.M. Mestechkin, and G.M. Zhidomirov, Theor. Exp. Chem. USSR 23, 123 (1987) (English translation).

[14] G.T. Klimko, M. M. Mestechkin, B. N. Plakhutin, G. M. Zhidomirov, and R. A. Evarestov, Int. J. Quantum Chem. 37, 35 (1990). 
[15] J.C. Slater, Quantum Theory of Atomic Structure (McGraw-Hill, New York, 1960), Vols. I and II.

[16] W. D. Edwards and M.C. Zerner, Theor. Chim. Acta 72, 347 (1987).

[17] C. J. Ballhausen, Introduction to Ligand Field Theory (McGraw-Hill, New York, 1961).

[18] H. P. Figeys, P. Geerlings, and C. Van Alsenoy, Int. J. Quantum Chem. 11, 705 (1977).

[19] M. Peterson and R. Poirier, MONSTERGAUSS-81 (Department of Chemistry, University of Toronto and Memorial University of Newfoundland, St. John's, Newfoundland, Canada).

[20] S. Huzinaga, J. Chem. Phys. 42, 1293 (1965).

[21] A. J. H. Wachters, J. Chem. Phys. 52, 1033 (1970).

[22] R. Poirier, R. Kari, and I. Csizmadia, Handbook of Gaussian Basis Sets, Physical Sciences Data. Vol. 24 (Elsevier, Amsterdam, 1985).

[23] E. Clementi and C. Roetti, At. Data Nucl. Data Tables 14, 177 (1974).

[24] C. Froese-Fisher, Compt. Phys. Commun. 4, 107 (1972); C. Froese-Fisher, Ibid. 7, 236 (1974).

[25] B. N. Plakhutin and A.V. Arbuznikov, to be submitted.

[26] J. B. Rose and V. McKoy, J. Chem. Phys. 55, 5435 (1971).

Received April 7, 1992

Revised manuscript received August 12, 1992

Accepted for publication September 6, 1992 